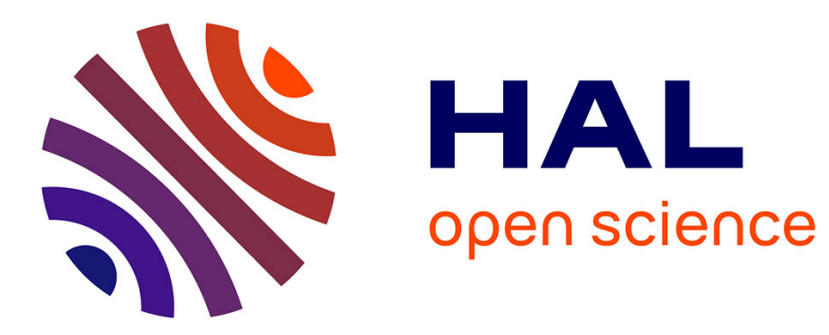

\title{
Numerical study, with experimental validation, of fan noise installation effects in Over-Wing Nacelle configuration using the Immersed Boundary Method
}

\author{
Mathieu Lorteau, Ludovic Wiart, Victor Kopiev
}

\section{- To cite this version:}

Mathieu Lorteau, Ludovic Wiart, Victor Kopiev. Numerical study, with experimental validation, of fan noise installation effects in Over-Wing Nacelle configuration using the Immersed Boundary Method. 25th AIAA/CEAS Aeroacoustics Conference, May 2019, DELFT, Netherlands. 10.2514/6.2019-2519 . hal-02196627

\section{HAL Id: hal-02196627 \\ https://hal.science/hal-02196627}

Submitted on 31 Jul 2019

HAL is a multi-disciplinary open access archive for the deposit and dissemination of scientific research documents, whether they are published or not. The documents may come from teaching and research institutions in France or abroad, or from public or private research centers.
L'archive ouverte pluridisciplinaire HAL, est destinée au dépôt et à la diffusion de documents scientifiques de niveau recherche, publiés ou non, émanant des établissements d'enseignement et de recherche français ou étrangers, des laboratoires publics ou privés. 


\title{
Numerical study, with experimental validation, of fan noise installation effects in Over-Wing Nacelle configuration using the Immersed Boundary Method
}

\author{
Mathieu Lorteau* and Ludovic Wiart ${ }^{\dagger}$ \\ Aerodynamics, Aeroelasticity and Acoustics Department, \\ ONERA - The French Aerospace Lab, \\ 29 avenue de la Division Leclerc, 92320 Châtillon, France \\ Victor Kopiev \\ Central Aerohydrodynamics Institute (TsAGI), Acoustic Department, \\ 17 Radio street, 105005 Moscow, Russia
}

\begin{abstract}
This work is part of the ONERA-TsAGI cooperative project NOWNA (NextGen Over the Wing Nacelle Aircraft) which investigates the potential of fan noise shielding for a transonic transport aircraft by installing a UHBR engine over its wing. The studied configuration is a derivative of the NOVA (Next Generation Onera Versatile Aircraft) for which cruise aerodynamic performance and low speed acoustics are evaluated and compared to a reference configuration with under wing engines. The present activity is a continuation of previous work on the numerical investigation of the acoustic advantages of Over the Wing Nacelle (OWN) configurations using ONERA's in-house CAA solver sAbrinA_v0 (see Mincu et al., AIAA 2017-3504). Whereas early computations did not assume any mean flow, the present work takes into account a realistic mean flow computed with ONERA's CFD solver FastS, at a global Mach number of 0.25 corresponding to take-off/landing flight conditions, and with a simple flow-through condition for the nacelle. Both CFD and CAA solvers use the Immersed Boundary Method (IBM) to deal with the realistic aircraft and nacelle geometries, which greatly simplifies the meshing stage. Here, this OWN configuration is compared to a more classic UWN (Under the Wing Nacelle) configuration, in terms of far field noise directivity.
\end{abstract}

\section{Introduction}

For many years, aircraft noise reduction has been the subject of research studies and continuous improvements were achieved through an improved understanding of the noise generation mechanisms. However, due to ever restrictive noise regulations around airports, new aircraft noise reduction strategies are mandatory. One strategy consists in studying new aircraft architecture such as OWN (Over the Wing Nacelle) aircraft. This type of architecture presents many potential benefits, such as fewer risks of debris ingestion from the runway and a full span slat; and also acoustic benefits: acoustic shielding and lower airframe noise, due to a shorter landing gear and weaker jet/flap interaction.

This work will focus on the fan noise installation effects of OWN configurations. To this aim, we will pursue the work initiated by Mincu et al. [1] who demonstrated, with a simplified source model and without any mean flow, the feasibility of numerical studies of installation effects considering a realistic aircraft geometry using the Immersed Boundary Method (IBM). This method gives a simplified way to take into account the effects of not only the solid walls but other boundary conditions and thus enables to overcome the mesh design difficulties inherent to complex geometries. This methodology is particularly well designed for parametric studies such as looking for an optimal position of a nacelle in OWN configuration in terms of acoustic shielding.

In the present work, we propose to use the IBM workflow implemented in the CAA solver sAbrinA_v0 [2-6] to study the installation effects in OWN configuration of the fan noise taking into account the aircraft and nacelle geometries in presence of a realistic mean flow at a Mach number $M=0.25$ corresponding to take-off / landing flight conditions. To this aim, we compare the acoustic far field directivity of the OWN configuration and the more classic

\footnotetext{
* Research engineer, mathieu.lorteau@ onera.fr

${ }^{\dagger}$ Research engineer, ludovic.wiart@onera.fr

$\ddagger$ Professor, Head of Acoustics Department, AIAA Senior Member
} 
UWN configuration. Both directivities are obtained from the same methodology which consists in first computing a realistic mean flow around the geometry under consideration. The mean flow is then interpolated on the CAA grid and used afterwards in the CAA computation in which we consider a simplified fan/OGV interaction noise model to compute the acoustic near field. This pressure field then serves as an input to compute the acoustic far field using the Ffowcs Williams \& Hawkings integral formulation [7, 8].

This paper is organized as follows. Section II presents the simulation parameters used in this work: the configuration of interest as well as the CFD and CAA solvers used in this work and finally the meshes (CFD and CAA) on which the simulations were performed. In section [II] the preliminary results of our study are presented. The ongoing developments provided in section [V] will be included in the final paper.

\section{Simulation parameters}

\section{A. Studied configuration}

The baseline UWN configuration was designed in a previous project focused on UHBR engine integration [9] using CFD calculations to minimize installation drag, see figure 1.

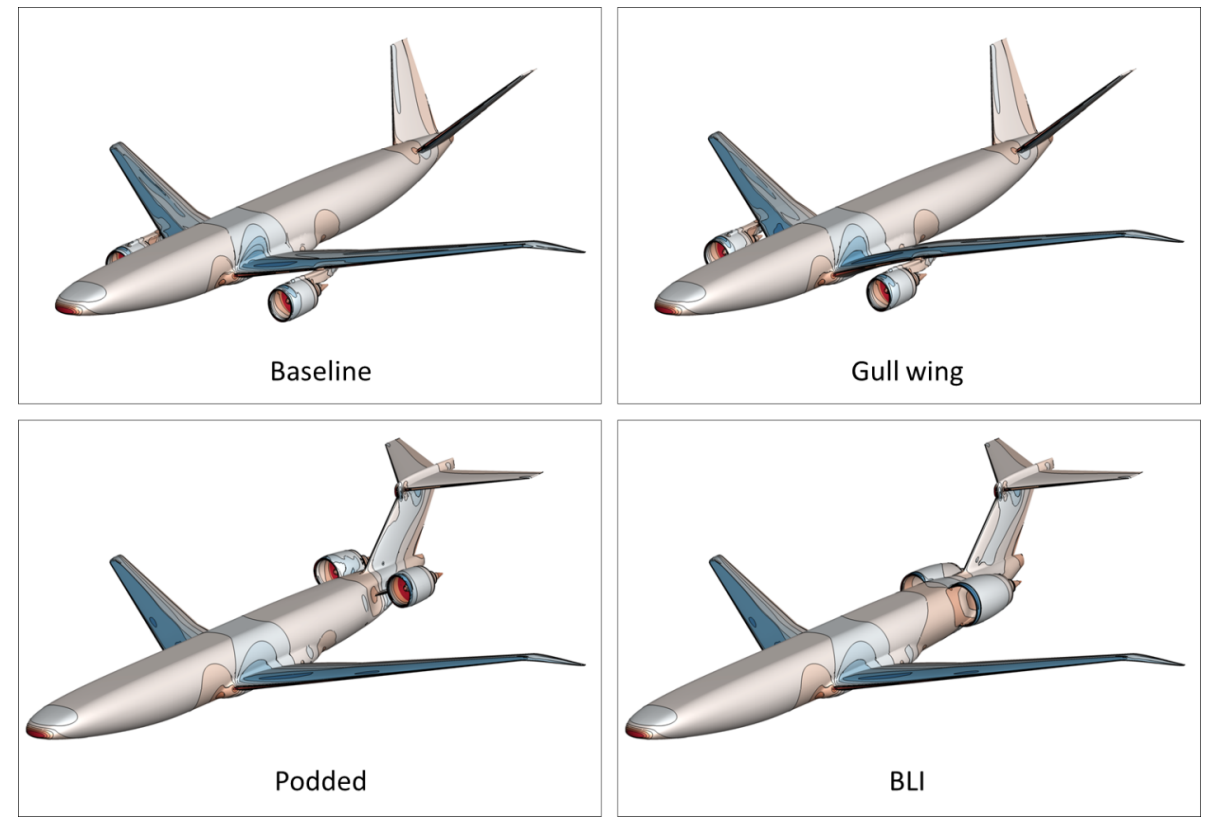

Fig. 1 General view of the NOVA configurations

The Reynolds Averaged Navier-Stokes (RANS) computations were performed with the elsA CFD solver [10]. This software uses a cell-centred Finite-Volume discretization on multi-block structured point-matched and overset meshes. Time integration is carried out by a backward-Euler scheme with implicit LU-SSOR relaxation. Spatial discretization is achieved using the $2^{\text {nd }}$ order centred scheme of Jameson et al. [11]. Multigrid techniques are used to accelerate the convergence to the steady solution. In this study, turbulence effects are simulated by the one equation Spalart-Allmaras model [12].

Since the NOVA configurations are meant to become reference research wind-tunnel models, all the aerodynamic calculations are performed in ONERA S1MA conditions $\left(P_{i}=90000 \mathrm{~Pa}, T_{i}=310 \mathrm{~K}\right)$ on a $1 / 9.35$ scale model at the cruise Mach number of 0.82 .

An engine placement study was performed. If it was fixed conventionally in the spanwise direction (just before the wing kink), the influence of vertical and axial position on aerodynamic performance was thoroughly investigated. The use of overlapping grids allows to move the engine around the aircraft without having to regenerate the whole mesh. While the ideal position of the engine in terms of aerodynamics is far away from the wing, the other critical constraint is the pylon weight, so that the selected position is necessarily a compromise.

A similar engine placement study was carried out with the OWN configuration, see figure 2 . However, the impact 
on aerodynamic performance was found to be greater in the OWN case due to the nacelle position effect on the upper wing shock pattern, in particular the lateral position. It was found that a very inboard position limits the installation drag penalty, in addition to being an advantage in terms of vertical tail sizing in case of engine failure.

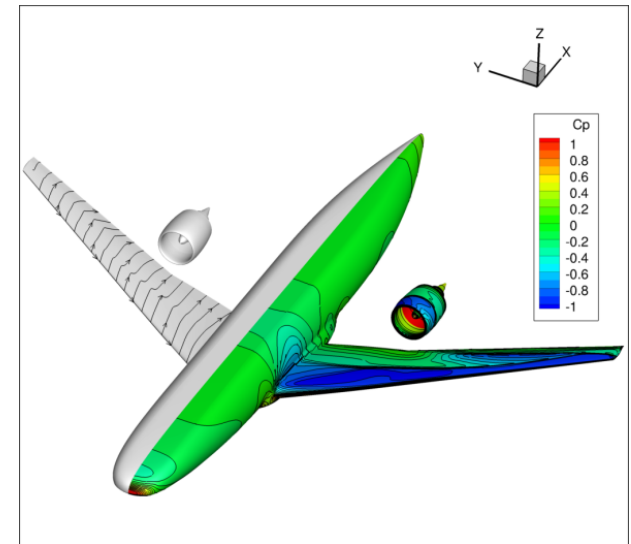

(a) Most outboard studied position

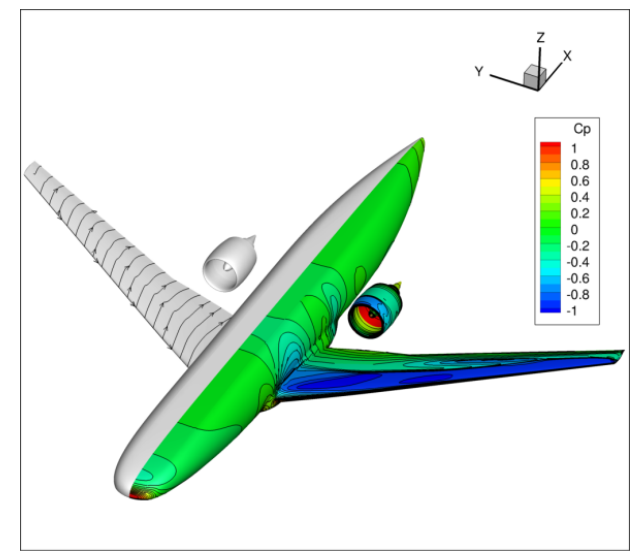

(c) Selected OWN position

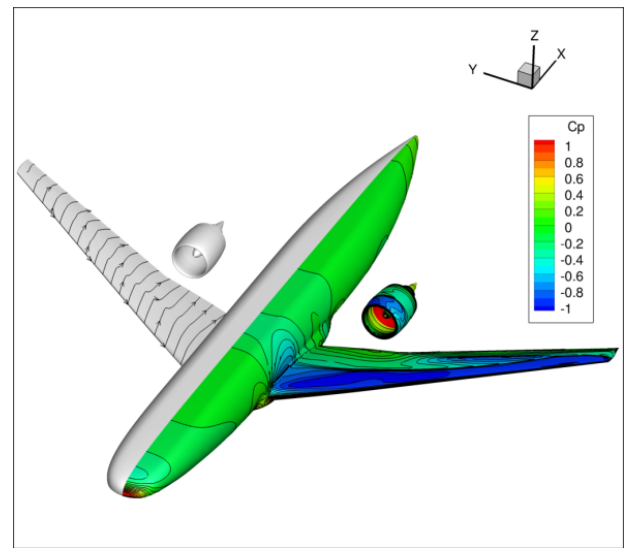

(b) Intermediary position

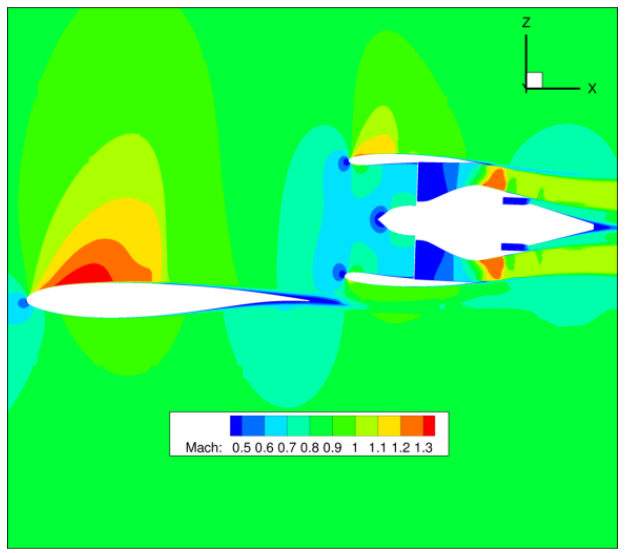

(d) Mach field in engine midsection plane in OWN position

Fig. 2 Effect of engine position on aerodynamic field $\left(C_{L}=0.5\right.$, Mach $\left.=0.82\right)$

While a pylon geometry is already available for the NOVA baseline UWN configuration, the design work is still ongoing on the OWN version. While its impact will have to be evaluated on cruise aerodynamic performance to fairly compare the UWN and OWN configurations, its effect on fan noise is expected to be low.

\section{B. Solvers and numerical parameters}

\section{CFD solver: FastS}

A CFD demonstrator named FastS has been developed at ONERA for several years [13-15] to provide a software architecture and numerical techniques allowing for a high level of efficiency, flexibility and upgradeability. This demonstrator is made by a set of independent modules, each of them defining a CFD solver dedicated to Cartesian, curvilinear and polyhedral grids, which rely on the CGNS/Python data representation. These modules work in an interoperable way with other modules, most of them being Cassiopee [16] modules devoted to pre-, co- and post-processing. Each component acts through Python functions on the same shared data (CGNS/Python tree in memory). Interior points of a Cartesian/structured/polyhedral grid are updated by the corresponding dedicated solver. A function enables to perform Chimera transfers between donor and receptor cells within Connector module of Cassiopee. This function has been generalized to handle also the update of IB (Immersed Boundary) target points, since the first step consists in a kind of Chimera interpolation, but for the IB image points. More details on the IBM implemented in Fast $S$ are available in Péron et al. [14]. Each CFD solver solves the compressible Euler and Navier-Stokes equations using a second-order accurate Finite-Volume Method only for the interior points of each grid. 
In this paper, the Cartesian solver of FastS is used to perform RANS simulations of the flow around the aircraft and the nacelle. In a first step, we have a simple flow-through condition for the nacelle, as the fan/OGV stage is not modelled. In the final paper, the actuator-disk or Body-Force method, for instance, will be used for this purpose. The turbulence modeling is handled by the Spalart-Allmaras model [12]. The Roe-MUSCL scheme is used with a first-order accurate implicit time integration and a local time step. The simulated flow is at a global Mach number of 0.25 corresponding to take-off/landing flight conditions.

\section{CAA solver: $s A b r i n A \_v O$}

The CAA calculations are conducted with Onera's $s A b r i n A \_v 0$ solver [2-6]. $s A b r i n A \_v 0$ is a structured grid, time-accurate CAA code that solves either the full or the linear Euler equations, in a conservative and perturbed form (with a splitting of the complete variables into a "frozen" mean flow and a "fluctuating" perturbation). The solver employs high-order, finite difference operators, involving 6th-order spatial derivatives and 10th-order filters, as well as a 3rd-order, multi-stage, Runge-Kutta time-marching scheme. The code deals with multi-block structured grids with one-to-one interfaces, and is fully parallelized using the Message Passing Interface (MPI) standard. Finally, the solver includes the usual boundary conditions (reflection by solid walls, non-reflecting / free-field radiation [17], etc.), as well as some unique to specific applications (such as the surface coupling technique [18, 19]). More detailed information about the $s A b r i n A \_v 0$ solver and its underlying methodology can be found in Ref. [20, 21].

The IBM implemented in $s A b r i n A \_v 0$ are based on the same numerical environment as those implemented in Fast $S$ in order to benefit from mutual developments. The geometrical features required for the IBM process are provided by functions of ONERA's Cassiopee module [16]. The developments specific to aeroacoustics issues are detailed in Mincu et al. [1].

The aeroacoustic simulations are performed with the linearised Euler equations, the geometries of the aircraft and nacelle (see section II.A) are taken into account with the IBM approach mentioned above. Following preliminary work on the same configuration presented in [1] but without any mean flow, we use in the present CAA simulation the realistic mean flow computed with the CFD solver FastS (see previous section). The flow field obtained on the CFD grid is interpolated on the CAA grid by the use of the in-house Cassiopee tool [16]. The acoustic source representing a fan/rotor interaction noise with an azimuthal mode $m=5$ at a frequency $f=8 \mathrm{kHz}$ is represented by a simplified model consisting of 60 monopoles located inside the nacelle at $90 \%$ of the nacelle inner radius. In the final paper, a more advanced model such as the one proposed by Polacsek et al. [3] will be used to simulate more acoustic modes in one computation, one of the advantage of the temporal approach.

\section{Grid parameters}

It was decided for this study to consider the full aircraft geometry with only one engine. The purpose is to focus on only one engine without the influence of the other one.

\section{CFD grid}

The mesh used for the CFD computation is composed by a set of uniform Cartesian grids, based on a skeleton defined by an octree [22]. Different levels of refinements are set near each obstacle. Cartesian grids overset with a minimum overlapping, such that information between domains is achieved by general Chimera transfers. The octree mesh is first built, with the finest refinement level located near the obstacle and fills the whole computational domain. Each element of the octree is then filled with a Cartesian grid of a user-specified number of cells per direction, which are then extended at their borders to overlap such that two layers of cells can be interpolated. In order to reduce the number of cells, grids that are entirely inside the solid are removed. Here the mesh is composed of $159.10^{6}$ cells with 856 grids with a minimum spacing $\Delta \approx 0.01 D_{n}$ (with $D_{n}$ the nacelle diameter) around the nacelle and the aircraft wing. Figure 3 gives an illustration of the mesh used for the aerodynamic computation, in particular the different mesh resolutions with the finest being around the nacelle and the wing next to it.

\section{CAA grid}

As mentioned in section III.B.2, in the CAA computation the geometry of interest is taken into account with the IBM approach, which thus greatly simplifies the CAA mesh design process for such a geometry. The mesh consists in a single block Cartesian grid. The zone of interest of our study corresponds to the zone located around the nacelle 


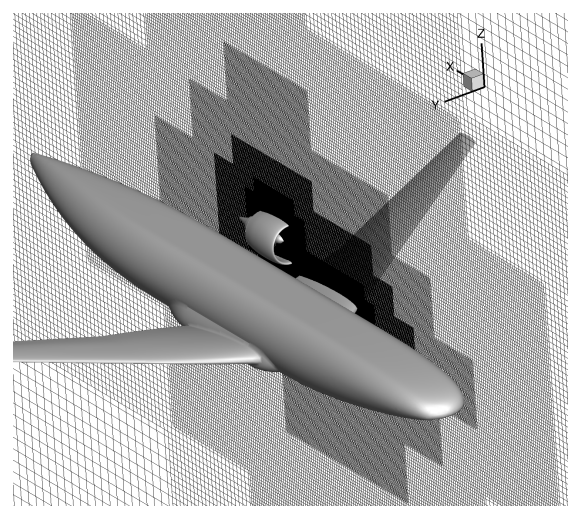

Fig. 3 Slice of the mesh used for the CFD computation with the NOVA aircraft and nacelle in OWN configuration

including the parts of the fuselage where the acoustic waves impact. In this zone, the mesh was defined with an isotropic cell size $\Delta=4 \cdot 10^{-3} \mathrm{~m}$. This cell size allows to resolve a frequency of $8 \mathrm{kHz}$ with $11 \mathrm{ppw}$ (points per wavelength) at rest and between 8 and $13 \mathrm{ppw}$ at $M=0.25$ (depending if we are upstream or downstream of the source respectively). The refined mesh zone boundaries are represented in red in figures 4 (a) and 4 (b). Starting from the refined mesh zone boundaries, the grid is stretched with a geometrical growth of $5 \%$ to reach 10 times the refined mesh size to the computational domain boundaries. This allows for the acoustic waves dissipation before reaching the boundaries and avoid pollution by reflected waves. In figure $4(\mathrm{a})$, the CAA computational domain boundaries are represented by the light blue box. Taking these considerations into account, the full mesh is composed of $472 \times 310 \times 235 \approx 34.10^{6}$ grid points.

The time step is $\Delta t=6.25 \times 10^{-6} \mathrm{~s}$ giving a maximum value of the CFL number about 0.7 . With these chosen parameters, we have computed the statistics on 1600 iterations corresponding to 16 rotations in about 1.25 hour on 300 MPI process, after a transient period of 2000 iterations. As a reference, the IBM preprocessing was performed in less than $3 \%$ of the statistics computation time.

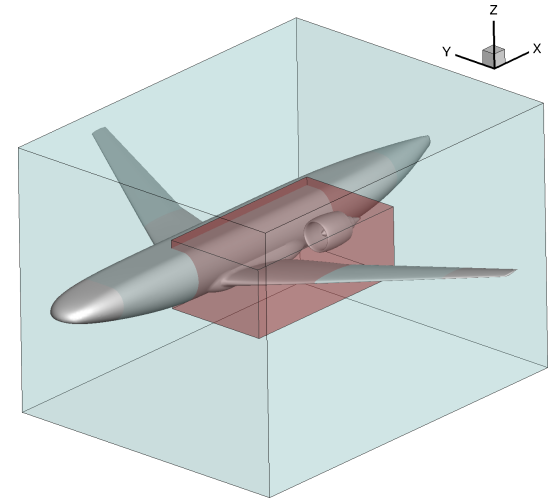

(a)

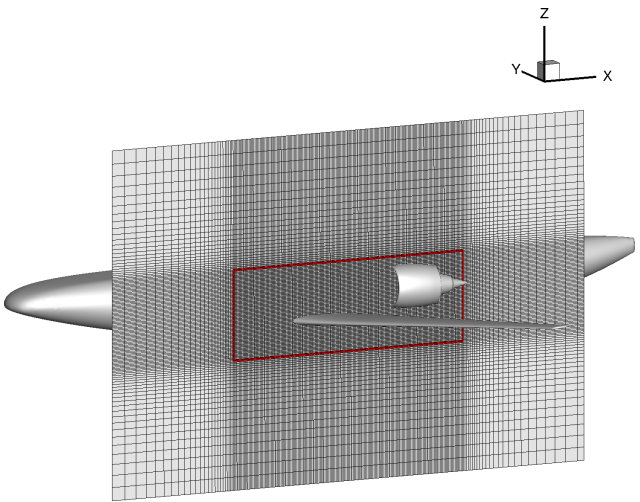

(b)

Fig. 4 Illustrations of the CAA computational domain (a) and the mesh in the nacelle midsection plane (b) with the refined mesh zone delimited in red

\section{Simulation results}

As of now, only preliminary results are available for the OWN configuration. The UWN configuration will be dealt with for the final paper (aerodynamic and aeroacoustic simulations and far field acoustic directivity computation). The acoustic benefit of the OWN configuration will then be studied through comparison of far-field directivities. 


\section{A. CFD flow}

Figure 5 represents a 2D map of the axial velocity field in the nacelle midsection plane for the OWN configuration. It appears that the mean flow is not properly converged yet and flow-through condition around the nacelle is evidenced. Nonetheless, this flow field is used for the CAA simulation in order to confirm the feasibility of the method. In the final paper, a properly converged mean flow field will be computed and used for the CAA computations.

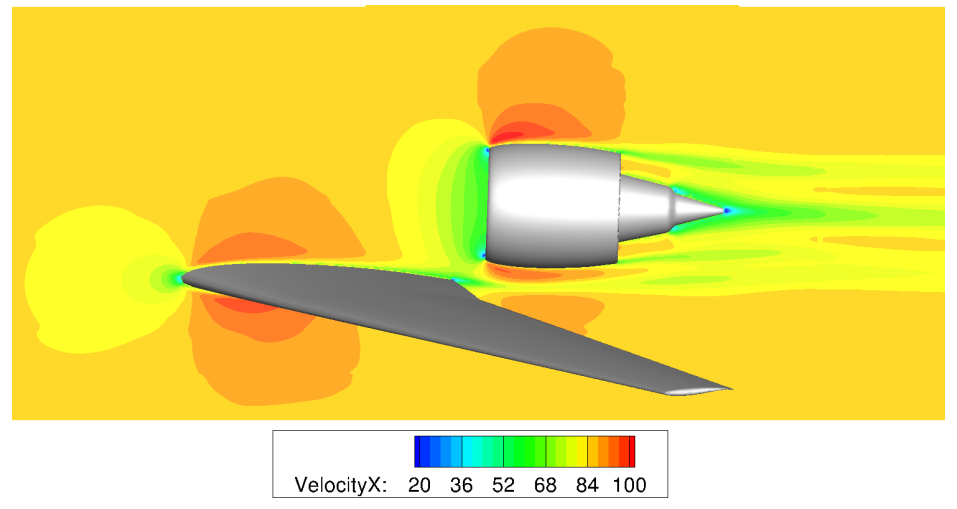

Fig. 5 Axial velocity field in nacelle midsection plane for OWN configuration

\section{B. Acoustic results}

In figure 6, the instantaneous fluctuating pressure field is represented in the refined mesh zone for the OWN configuration. We can see that the acoustic waves are radiated at around $45^{\circ}$ from the nacelle axis which impacts on the wing thus giving a shielding effect. The low pressure levels under the wing are an illustration of it.

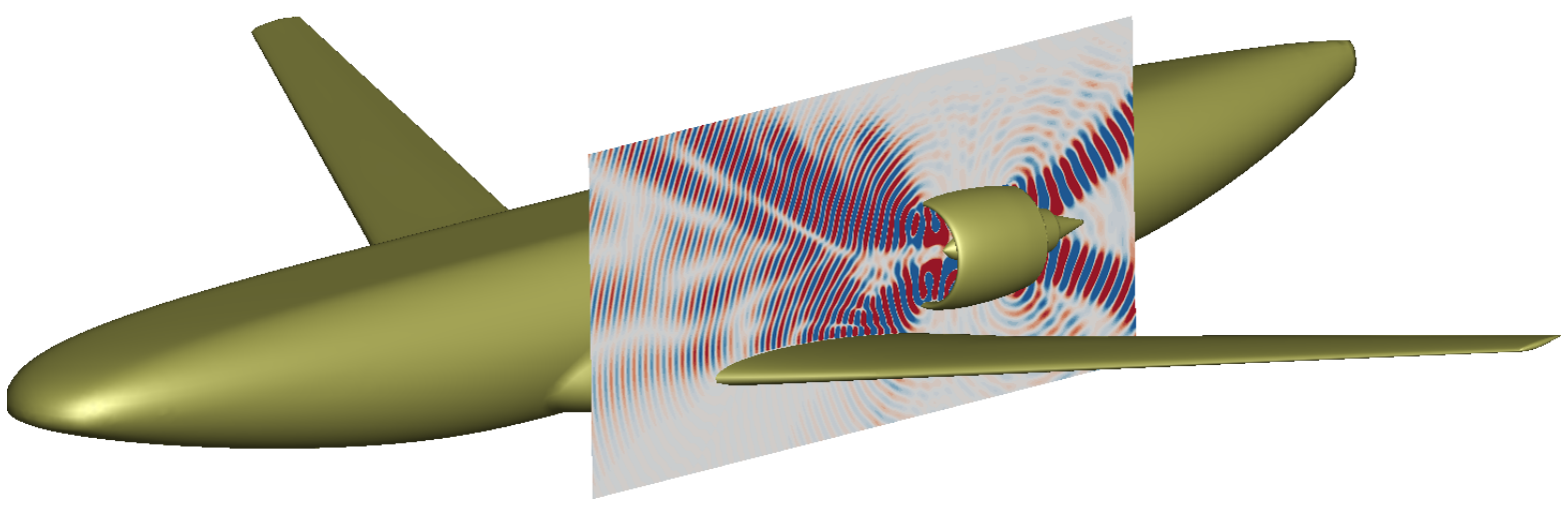

Fig. 6 Instantaneous fluctuating pressure field in the nacelle midsection plane for OWN configuration

In the following, we plan to simulate the UWN configuration with the same methodology. We will then quantify the shielding effect through comparisons of the far-field pressure levels (obtained with Ffowcs-Williams \& Hawkings [7, 8] integral formulation) and cartographies of the acoustic impact on the ground taking into account the aircraft trajectory.

\section{Perspectives}

The preliminary results presented above will be completed by the installation effects in Over-Wing Nacelle configuration quantification. For this, the same methodology will be applied to the more classic UWN configuration. Moreover, to obtain an even more realistic simulated configuration, a mean flow taking into account the fan/OGV stage (e.g. body-force method) and the pylon joining the nacelle and the wing will be computed. This will influence the propagation of the acoustic mode, downstream of the nacelle for the fan/OGV stage modelling and upstream of the nacelle for the pylon. To make use of the full potential of the temporal approach of the solver 
sAbrinA_v0, more realistic fan noise source terms [3, 5] will be used in order to compute several acoustic modes at the same time, which we can study separately afterwards through Fourier analysis. The multimodal simulations will require longer simulated time for convergence purpose. In the framework of the NOWNA cooperation, experimental measurements of the shielding effect of a simplified wing model have been performed at rest. The already available database will give an interesting reference for the numerical study and will be included in the final paper.

\section{Acknowledgments}

This work has been made within the framework of the NOWNA cooperative project between ONERA and TsAGI. The authors would like to thank Ivan Mary, Christophe Benoit and Thomas Renaud for their precious help with FastS solver and Cassiopee modules. Thomas Le Garrec and Daniel-Ciprian Mincu are gratefully acknowledged for their support with the $s A b r i n A \_v 0$ solver.

\section{References}

[1] Mincu, D. C., Le Garrec, T., Peron, S., and Terracol, M., "Immersed boundary conditions for high order CAA solversAeroacoustics installation effects assessment," 23rd AIAA/CEAS Aeroacoustics Conference, 2017.

[2] Terracol, M., Manoha, E., Herrero, C., Labourasse, E., Redonnet, S., and Sagaut, P., "Hybrid method for airframe noise numerical prediction," Theoretical and Computational Fluid Dynamics, Vol. 19, 2005, pp. 197-227.

[3] Polacsek, C., Desquesnes, G., and Reboul, G., "An equivalent-source model for simulating noise generation in turbofan engines," Journal of Sound and Vibration, Vol. 323, 2009, pp. 697-717.

[4] Redonnet, S., Desquesnes, G., Manoha, E., and Parzini, C., "Numerical study of acoustic installation effects with computational aeroacoustics method," AIAA Journal, Vol. 48, No. 5, 2010, pp. 929-937.

[5] Reboul, G., and Polacsek, C., "Towards Numerical Simulation of Fan Broadband Noise Aft Radiation from Aero-engines," AIAA Journal, Vol. 48, No. 9, 2010, pp. 2038-2048.

[6] Redonnet, S., and Druon, Y., "Computational Aeroacoustics of Realistic Co-Axial Engines," AIAA Journal, Vol. 50, No. 5, 2012, pp. 1029-1046.

[7] Ffowcs Williams, J. E., and Hawkings, D. L., "Sound generation by turbulence and surfaces in arbitrary motion," Philosophical Transactions of the Royal Society of London A, Vol. 264, No. 1151, 1969.

[8] Rahier, G., Prieur, J., Vuillot, F., Lupoglazoff, N., and Biancherin, A., "Investigation of integral surface formulations for acoustic post-processing of unsteady aerodynamic jet simulations," Aerospace Science and Technology, Vol. 8, 2004.

[9] Wiart, L., Atinault, O., Grenon, R., Paluch, B., and Hue, D., "Development of NOVA Aircraft Configurations for Large Engine Integration Studies," 33rd AIAA Applied Aerodynamics Conference, Vol. (2254), 2015.

[10] Cambier, L., Heib, S., and Plot, S., "The Onera elsA CFD software: input from research and feedback from industry," Mechanics \& Industry, Vol. 14, No. 3, 2013, pp. 159-174.

[11] Jameson, A., Schmidt, W., and Turkel, E., "Numerical Solution of the Euler Equations by Finite Volume Methods Using Runge Kutta Time Stepping Schemes,” 14th Fluid and Plasma Dynamics Conference, Vol. (1259), 1981.

[12] Spalart, P. R., and Allmaras, S. R., “A one-equation turbulence model for aerodynamic flows,” 30th Aerospace Sciences Meeting and Exhibit, Vol. (439), 1992.

[13] Mary, I., "Flexible Aerodynamic Solver Technology in an HPC environment," Maison de la Simulation Seminars, Vol. http://www.maisondelasimulation.fr/seminar/data/201611 slides 1.ppt, 2016.

[14] Péron, S., Renaud, T., Terracol, M., Benoit, C., and Mary, I., "An immersed boundary method for preliminary design aerodynamic studies of complex configurations," 23rd AIAA Computational Fluid Dynamics Conference, Vol. (3623), 2017.

[15] Dandois, J., Mary, I., and Brion, V., "Large-eddy simulation of laminar transonic buffet," Journal of Fluid Mechanics, Vol. 850, 2018, pp. 156-178.

[16] Benoit, C., Péron, S., and Landier, S., "Cassiopee: A CFD pre- and post-processing tool," Aerospace Science and Technology, Vol. 45, 2015, pp. $272-283$. 
[17] Guenanff, R., "Couplage instationnaire Navier-Stokes/Euler pour la génération et le rayonnement des sources de bruit aérodynamique," Ph.D. thesis, Université Rennes 1, 2004.

[18] Redonnet, S., "On the numerical prediction of aerodynamic noise via a bybrid approach. Part 1: CFD/CAA surfacic coupling methodology, revisited for the prediction of installed airframe noise problems," AIAA paper, Vol. (3709), 2010.

[19] Redonnet, S., Lockard, D., Khorrami, M., and Choudhari, M., "CFD-CAA Coupled Calculations of a Tandem Cylinder Configuration to Assess Facility Installation Effects,” AIAA paper, Vol. (2841), 2011.

[20] Redonnet, S., Manoha, E., and Sagaut, P., "Numerical simulation of propagation of small perturbations interacting with flows and solid bodies," AIAA paper, Vol. (3493), 2001.

[21] Redonnet, S., "Simulation de la propagation acoustique en présence d'écoulements quelconques et de structures solides, par résolution numérique des équations d'Euler,” Ph.D. thesis, Université Bordeaux 1, 2001.

[22] Péron, S., and Benoit, C., "Automatic off-body overset adaptive Cartesian mesh method based on an octree approach," Journal of Computational Physics, Vol. 232, No. 1, 2013, pp. 153-173. 